\section{Transvaginal compared with transvesical ultrasonography for recovery of oocytes for in vitro fertilisation}

Oocyte recovery guided by transvaginal ultrasonography has been introduced into in vitro fertilisation units and compares favourably with laparoscopic oocyte recovery. ${ }^{12}$ The transition from laparoscopy to ultrasonography may, however, account for part of this difference-for example, anaesthetic requirements and risks of pneumoperitoneum are reduced. In Oxford the in vitro fertilisation unit is an outpatient service and used transvesical ultrasonography ${ }^{3}$ until transvaginal scanning was introduced. Thus we were able to make a direct assessment of the value of oocyte recovery by transvaginal scanning.

\section{Patients, methods, and results}

We report the results of 100 consecutive cycles of in vitro fertilisation. There was a definite transition between the final 50 women, undergoing transvesica ultrasonography and the first 50 women undergoing transvaginal ultrasonography (February-March 1987 and April-June 1987, respectively). The date when oocyte recovery by vaginal scanning was to be introduced was not known when the cycles of treatment were booked, so clinical factors did not affect the composition of the groups. Clinical and laboratory methods were unchanged over both periods. This unit uses norethisterone to schedule cycles and a fixed stimulation regimen to predetermine the day of oocyte recovery. ${ }^{+}$Thus any differences in outcome cannot be attributed to subjective decision making about the timing of oocyte recovery, except in a few cycles in each group in which buserelin was used to desensitise the pituitary (transvesical scanning, seven; vaginal scanning, four). An ATL 100 scanner with $3.5 \mathrm{MHz}$ sector probe and needle guide was used in transvesical scanning; a Phillips R1550 XP S scanner with a $5 \mathrm{MHz}$ vaginal probe fitted with the Phillips smooth contour needle guide was used in transvaginal scanning. All recoveries of oocytes were performed with 17 gauge needles, the women being sedated only with intravenous pethidine and diazepam. The policy of the unit is to aim to transfer a maximum of three embryos, with exceptionally four embryos transferred when four poor quality embryos have resulted. In this series six women received four embryos (transvesical scanning, two; vaginal scanning, four)

The groups were similar clinically and in their responses to stimulation (table) The numbers of oocytes recovered and fertilised per recovery were significantly higher with transvaginal than transvesical ultrasonography $(p<0.0001$ for both cases Spearman's rank coefficient). The number of embryos transferred was significantly different between the two groups $(p<0.0001$, Spearman's rank coefficient), so, although the rate of clinical pregnancy in women receiving more than two embryos was not different between the two groups (transvesical scanning, 36\%; transvaginal scanning, 39\%), the proportion of the group undergoing transvaginal scanning who received more than two embryos was

Clinical details of and results in women undergoing oocyte recovery by transvesical or transvaginal ultrasonography

Vesical scanning Vaginal scanning

\begin{tabular}{|c|c|c|}
\hline & Vesical scanning & Vaginal scanning \\
\hline \multicolumn{3}{|l|}{ Clinical details } \\
\hline Mean age (range) (years) & $32 \cdot 7(25-40)$ & $31 \cdot 6(22-42)$ \\
\hline Mean duration of infertility (range) (years) & $5 \cdot 0(2-12)$ & $5.9(1-14)$ \\
\hline \multicolumn{3}{|l|}{ No (\%) with infertility factor: } \\
\hline Tubal & $41(82)$ & $42(84)$ \\
\hline Male & $9(18)$ & $5(10)$ \\
\hline Endometriosis & $4(8)$ & $3(6)$ \\
\hline Unexplained & $9(18)$ & $8(16)$ \\
\hline \multicolumn{3}{|l|}{ On day of stimulation with $\mathrm{HCG}^{\star}$ : } \\
\hline Median plasma oestradiol (range) (pmol/l) & $3290(833-14430)$ & $3090(648-15170)$ \\
\hline Median plasma progesterone (range) $(\mathrm{nmol} / \mathrm{l})$ : & $0 \cdot 6(0 \cdot 3-13 \cdot 4)$ & $1 \cdot 3(0 \cdot 3-7 \cdot 3)$ \\
\hline $\begin{array}{l}\text { No }(\%)>6 \mathrm{nmol} / 1 \\
\text { Median fiameter ofleading follicle (range) (mm) }\end{array}$ & $4(8)$ & $3(6)$ \\
\hline Median diameter of leading follicle (range) $(\mathrm{mm})$ & $18(14-28)$ & $17(11-25)$ \\
\hline \multicolumn{3}{|l|}{ Results } \\
\hline No $(\%)$ of cycles started & $50(100)$ & $50(100)$ \\
\hline No $(\%)$ of oocyte recoveries performed & $48(96)$ & $48(96)$ \\
\hline No $(\%)$ with at least one oocyte & $45(90)$ & $47(94)$ \\
\hline Median no of oocytes/recovery (range) & $3(0-12)$ & $4(0-10) t$ \\
\hline Median no of oocytes fertilised/recovery (range) & $1(0-5)$ & $2(0-7) \dagger$ \\
\hline Median no of embryos transferred (range) & $1(0-4)$ & $2(0-4) \dagger$ \\
\hline No (\%) with 1 or 2 embryos transferred & $20(40)$ & $16(32)$ \\
\hline No (\%) with $>2$ embryos transferred & $11(22)$ & $23(46) \dagger$ \\
\hline No $(\%)$ of embryo transfers & $31(62)$ & $39(78)$ \\
\hline \multicolumn{3}{|l|}{ Clinical pregnancies: } \\
\hline No & 6 & 12 \\
\hline Rate per cycle (\%) & 12 & $24 \dagger$ \\
\hline Rate per embryo transfer (\%) & 19 & $31 \dagger$ \\
\hline
\end{tabular}

${ }^{\star} \mathrm{HCG}=$ Human chorionic gonadotrophin

†Significant differences (see text) significantly higher $(p<0.01$, Fisher's exact test; transvesical scanning, 22\%, transvaginal scanning, $46 \%$ ). The rate of clinical pregnancy per cycle was doubled, in keeping with the higher yield of oocytes and embryos, in the group undergoing transvaginal scanning $(\mathrm{p}<0 \cdot 01$, Fisher's exact test).

\section{Comment}

The attraction of transvaginal scanning is that it provides more detailed imaging and removes the undoubted distress and unpredictable delays to patients associated with techniques that require a full bladder. Our results confirmed expectations, and we immediately transferred to oocyte recovery by transvaginal scanning. Our initial experience produced results superior to our accumulated experience over about 300 cycles with transvesica scanning. The transvaginal method improved yields at each step of in vitro fertilisation, with a higher proportion of women receiving the optimal number of embryos and a higher proportion of cycles resulting in pregnancy. Since the introduction of transvaginal methods the low cost in vitro fertilisation unit in Oxford ${ }^{5}$ has achieved results comparable with those of leading centres, indicating that small units setting up in vitro fertilisation programmes should consider adopting transvaginal methods from the outset.

1 Torode HW, Picker RH, Porter RN, et al. Oocyte pickup by laparoscopy replaced by transvaginal in vitro fertilisation program. Journal of In Vitro Ferilisation and Embryo Transfer 1987;4:148-52

2 Maleika F, Maleika MC, Hartter P. Engers G, Weiske WH. Comparison between trans-vaginal ultrasound and pelviscopic oocyte retrieval for IVF. Human Reproduction 1987;2 (suppl 1):54.

Little DJ, Bromwich PD, Walker AP, Macken AM, Newton JR. A simplified approach to oocyte recovery for in-vitro fertilization. Brf Obstet Gynaecol 1985;92:180-1.

4 Walker A, Bromwich P, Barlow DH, et al. A comparative study of fixed-schedule and individualised stimulation for in-vitro fertilisation. Human Reproduction 1987;2:(suppl 1):34.

5 Bromwich P, Walker A, Kennedy S, et al. In vitro fertilisation in a small unit in the NHS. Br Med $\mathcal{J}$ $1988 ; 296: 759$.

(Accepted 5 fanuary 1988

Nuffield Department of Obstetrics and Gynaecology, University of Oxford

John Radcliffe Hospital, Headington, Oxford OX3 9DU

DAVID BARLOW MD, MRCOG, clinical reader

PETER BROMWICH, MRCOG, clinical lecture

MARY WILEY, BSC, SCM, research nursing sister

ANDREW WALKER, BSC, scientist

CAROLINE ROSS, BSC, scientist

STEPHEN KENNEDY, $M A, M B$, clinical research fellow

ANDRES LOPEZ-BERNAL, LMS, DPHIL, clinical research fellow

Correspondence and requests for reprints to: $\mathrm{Mr}$ Barlow.

\section{Effect of high dose somatostatin analogue on growth hormone concentrations in acromegaly}

Octreotide, a somatostatin analogue, given subcutaneously successfully reduces growth hormone concentrations in acromegaly, but not to within the normal range $(<5 \mathrm{mIU} / \mathrm{l}) \cdot{ }^{1.5}$ Unfortunately, somatostatin also suppresses plasma insulin concentrations and can impair glucose tolerance. ${ }^{23}$ In view of this we compared the biochemical effects of increasing doses of octreotide in patients with acromegaly.

\section{Patients, methods, and results}

After their informed consent had been obtained six patients with active acromegaly were given octreotide $0 \cdot 3,1 \cdot 5$, or $3 \mathrm{mg}$ daily in divided doses by subcutaneous injection at 0800,1600 , and 2200 . A control profile was taken, and then the patients were given each dose for three days, during which blood was taken for measurement of growth hormone, insulin, and glucose concentrations at fixed times $(n=15)$ over 24 hours. Standard meals were provided on each tes day. In a long term study four of the patients continued to take octreotide $1000 \mu \mathrm{g}$ thrice daily for a mean of four months (range three to six).

The table shows the fall in 24 hour growth hormone and insulin concentrations with the three doses of octreotide compared with the pretreatment concentrations Compared with the lowest dose regimen, the highest dose caused a significant reduction in both variables; there was no other significant difference among the three doses. In addition, there was no significant change in mean glucose 
Mean (SEM) concentrations of growth hormone, insulin, and glucose over 24 hours with each dose regimen. For statistical analysis (Student's paired t test) growth hormone and insulin concentrations were log transformed

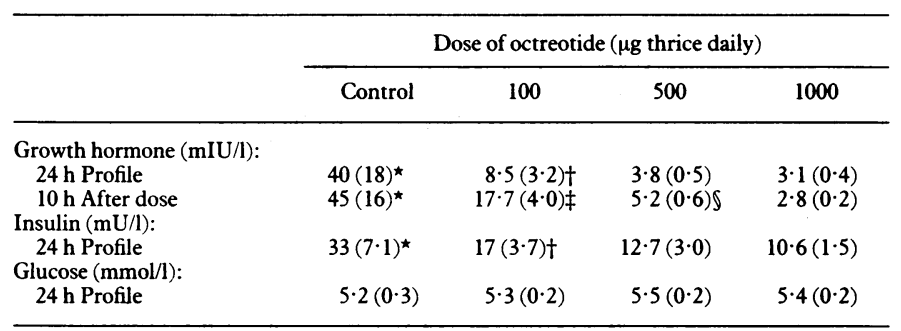

$\star$ Control $v 100,500$, and $1000 \mu \mathrm{g}<0.001$.

$+100 v 1000 \mu \mathrm{g} \mathrm{p}<0.05$.

$\neq 100 v 500 \mu \mathrm{g} \mathrm{p}<0.05$.

$\$ 500 v 1000 \mu \mathrm{g} p<0.02$.

concentration with any of the doses. Growth hormone concentration was within normal limits 10 hours after $1000 \mu \mathrm{g}$ octreotide but not after 100 or $500 \mu \mathrm{g}$. Daily measurements of growth hormone concentration showed no change on each of the three days of each dose regimen.

Twenty four hour profiles obtained in the four patients on the last day of the long term study did not differ from those obtained in the acute study at this dose.

No patient experienced subjective or objective side effects while taking octreotide. There was no change in blood count, biochemical profile, or thyroxine, cortisol, prolactin, luteinising hormone, follicle stimulating hormone, and sex hormone concentrations at the highest dose.

\section{Comment}

Several studies have indicated that octreotide is an effective treatment for acromegaly, although in the recommended dose (50-100 $\mu \mathrm{g}$ thrice daily) it is unsuccessful in suppressing growth hormone concentrations throughout the interval between two injections. ${ }^{25}$ Our data ${ }^{2}$ and those of others ${ }^{4}$ have shown that after $100 \mu \mathrm{g}$ subcutaneously suppression of growth hormone is maximal at two to four hours. Timsit et al reported that $500 \mu \mathrm{g}$ thrice daily given subcutaneously did not normalise growth hormone in one of two subjects studied. ${ }^{5}$ They indicated that continuous subcutaneous infusion of octreotide $(100 \mu \mathrm{g} / 24 \mathrm{~h})$ normalised growth hormone throughout the day in six of seven patients studied. Our study confirms that subcutaneous octreotide in doses of $1000 \mu \mathrm{g}$ thrice daily can normalise growth hormone concentration in acromegaly. Indeed, in all six patients in our study the mean 24 hour growth hormone concentration was normal with the highest dose used and there was no evidence that growth hormone concentration increased between doses, as it did with the 100 and $500 \mu \mathrm{g}$ doses.

It has been reported that octreotide may worsen glucose tolerance in acromegaly by suppressing plasma insulin concentrations. ${ }^{23}$ Our study confirmed that the reduction in insulin concentration was greater with 1000 $\mu \mathrm{g}$ than $100 \mu \mathrm{g}$ thrice daily. Nevertheless, in both the short and long term studies there was no evidence of an appreciable deterioration in 24 hour plasma glucose concentration with any of the dose regimens, which might be due to a simultaneous greater reduction in counterregulatory factors enhancing insulin sensitivity. Previous studies of low dose subcutaneous octreotide have indicated that the drug is well tolerated. Although further long term data are necessary, our study suggests that higher doses are also without important side effects.

1 Ch'ng JLC, Sandler LM, Kraenzlin ME, Burrin JM, Joplin GF, Bloom SR. Long term treatment of acromegaly with a long acting analogue of somatostatin. $\mathrm{BrMed} \mathcal{F}$ 1985;290:284-5.

2 Sandler LM, Burrin JM, Williams G, Joplin GF, Carr DH, Bloom SR. Effective long-term treatment of acromegaly with a long-acting somatostatin analogue (SMS 201-995). Clin Endocrinol (Oxf) 1987;26:85-95.

3 Lamberts SW, Uitterlinden P, Verschoor L, van Dongen KJ, del Pozo E. Long-term treatment of acromegaly with the somatostatin analogue SMS 201-995. N Englf Med 1985;313:1576-80.

4 Comi RJ, Gorden P. The response of serum growth hormone levels to the long-acting somatostatin analog SMS 201-995 in acromegaly. F Clin Endocrinol Metab 1987;64:37-42.

5 Timsit J, Chanson P, Larger E, et al. The effect of subcutaneous infusion versus subcutaneous injections of a somatostatin analogue (SMS 201-995) on the diurnal GH profile in acromegaly. Acta Endocrinol (Copenh) 1987;116:108-12.

(Accepted 9 November 1987)

Department of Medicine, Royal Postgraduate Medical School, Hammersmith Hospital, London W12 0HS

L M SANDLER, MRCP, senior registrar

J M BURRIN, PHD, top grade biochemist

G F JOPLIN, PHD, FRCP, professor of clinical endocrinology

S R BLOOM, MD, FRCP, professor of endocrinology

Correspondence to: Dr Sandler.

\section{Dialysis myelopathy: quadriparesis due to extradural amyloid of $\beta_{2}$ microglobulin origin}

We report a case of cervical myelopathy due to extradural deposition of amyloid derived from $\beta_{2}$ microglobulin. Posterior cervical laminectomy resulted in considerable improvement.

\section{Case report}

A 47 year old West Indian man was admitted for investigation of a four month history of progressive stiffness and weakness of his arms and legs and numbness of the lower half of his body. He had started receiving haemodialysis in 1970. In 1978 he developed hyperparathyroidism necessitating parathyroidectomy. Subsequent dialysis arthropathy was confirmed by the presence of synovial amyloid derived from $\beta_{2}$ microglobulin in the right knee. In 1985 he received a cadaver renal transplant but developed chronic rejection, and he resumed haemodialysis nine months after transplantation. In 1986 biopsy of a cyst in the clavicle showed deposition of amyloid derived from $\beta_{2}$ microglobulin.

When admitted to hospital he was unable to walk, move from a chair to bed or hold a pen to write. Examination showed a spastic quadriparesis with pronounced rigidity, hyperreflexia, and extensor plantar reflexes. Joint position sense was reduced, and the response to pin prick was reduced below the upper thorax. Myelography showed posterior displacement of the thecae at the sacrum, L3-5, and T7. There was almost complete obstruction to flow of contrast medium from $\mathrm{T} 1$ to $\mathrm{C} 2$. Computed tomography of the cervical region showed widespread extradural soft tissue densities with almost total obliteration of the subarachnoid space at C5-6. The cord was noticeably compressed (figure).

Posterior cervical laminectomy was performed. The spinal cord was completely surrounded by concentric rings of yellow fibrous tissue, which was removed. Histological examination showed extensive extracellular deposits of amyloid within fibroelastic ligament. Immunoperoxidase stains were positive for $\beta_{2}$ microglobulin but negative for serum amyloid A proteins and $x$ and $\lambda$ light chains. Postoperative recovery was good. He could walk and climb stairs, and sensation returned to normal.

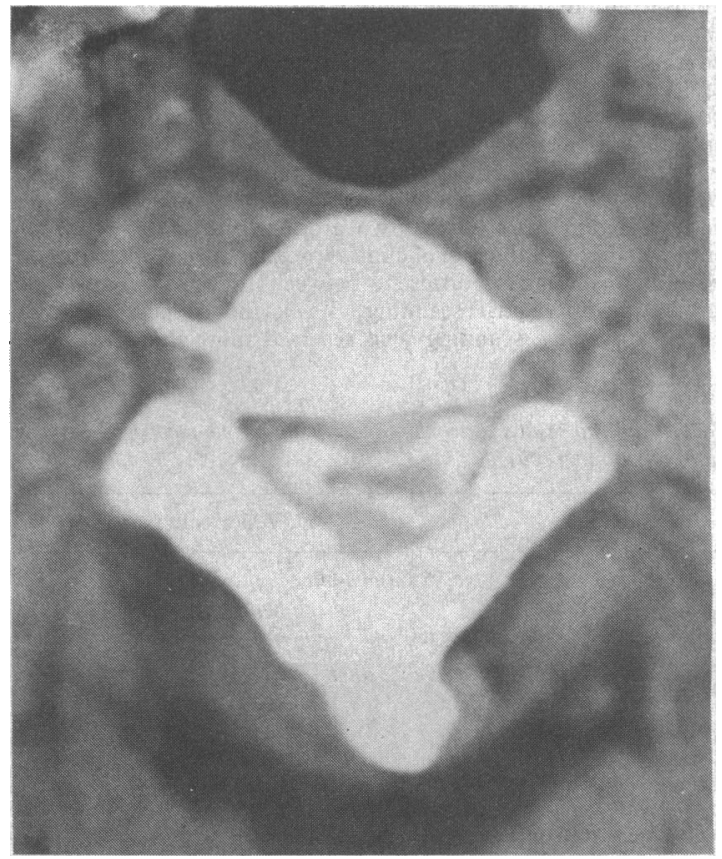

Computed tomogram of cervical cord at $\mathrm{C} 2-3$

\section{Comment}

Compression of the spinal cord due to extradural amyloid is rare. ${ }^{12}$ In previous cases no primary cause for the formation of amyloid was found, although monoclonal gammopathy was implicated in one report. ${ }^{2}$ This is the first reported case of compression of the cord due to extradural deposition of amyloid derived from $\beta_{2}$ microglobulin.

Dialysis arthropathy, bone cysts, and recurrent carpal tunnel syndrome due to deposition of amyloid are recognised complications of long term haemodialysis. ${ }^{3}$ Biochemical and immunohistochemical studies have identified the precursor molecule of the amyloid as $\beta_{2}$ microglobulin. $\beta_{2}$ Microglobulin concentrations are raised in patients undergoing dialysis 